\title{
Problemas de enfermería en el trasplante a pacientes añosos
}

\author{
Fernando Ramos Peña - Soraya de Cos Echaniz - M Plácida García Mota - Amparo Delgado Requejo \\ Leonor Ahedo Arrien - Ana Rosa Muñoz García - Carmen Gutiérrez García - Milagros Menchaca Casin
}

Servicio de Nefrología. Sección de Agudos. Hospital de Cruces. Baracaldo

\section{Resumen}

El objetivo de este trabajo fue identificar los problemas de enfermería en la atención a pacientes añosos (a partir de 65 años), receptores de injerto renal durante su ingreso hospitalario para el trasplante. Para ello se analizaron retrospectivamente las historias clínicas de los años 2005 y 2006, codificándose los problemas de enfermería mediante la Taxonomía NANDA. Se utilizó la estadística descriptiva para mostrar la frecuencia y duración de los problemas, así como la presencia de complicaciones médicas. También se contabilizaron el número de transfusiones sanguíneas, número de hemodiálisis post-trasplante y el número de pacientes que precisaron de suero de irrigación continua vesical para tratar los problemas de coágulos intravesicales. Los problemas de enfermería más frecuentes son: riesgo de infección, riesgo de lesión perioperatoria, exceso de volumen de líquidos, deterioro de la integridad cutánea, deterioro de la movilidad física, déficit de autocuidados, conocimientos deficientes y deterioro del patrón del sueño. Los problemas que, al alta, quedaron sin resolver de forma más frecuente fueron deterioro de la eliminación urinaria, conocimientos deficientes, riesgo de glucemia inestable y deterioro del patrón del sueño. Las complicaciones médicas más frecuentes fueron la necrosis tubular aguda y la fístula urinaria. El 23\% de los pacientes necesitaron transfusiones de sangre post-cirugía, el $45 \%$

\begin{tabular}{|c|}
\hline Correspondencia: \\
Fernando Ramos Peña \\
Servicio de Nefrología, Sección Agudos, 7ª Planta \\
Hospital de Cruces \\
Plaza de Cruces s/n \\
48903 Baracaldo \\
framos@euskalnet.net
\end{tabular}

necesitó de hemodiálisis en los días posteriores al trasplante y el $38 \%$ necesitó de irrigación continua vesical.

\section{PALABRAS CLAVE:}

- TRASPLANTE RENAL

PACIENTES AÑOSOS

COMPLICACIONES

TAXONOMÍA NANDA

\section{Nursing problems in transplants in elderly pa- tients}

\section{Abstract}

The aim of this work was to identify the nursing problems in the care of elderly patients (aged 65 and over) who receive a kidney graft during their time in hospital for transplant. To do so, the clinical histories of the years 2005 and 2006 were analysed retrospectively, and the nursing problems were coded using NANDA taxonomy. Descriptive statistics were used to show the frequency and duration of the problems, and the presence of medical complications. The number of blood transfusions, number of cases of post-transplant haemodialysis and the number of patients who required continuous vesicular irrigation to treat intravesicular clotting problems were also recorded. The most frequent nursing problems are: risk of infection, risk of perioperatory injury, excess of liquids, deterioration of skin integrity, deterioration of physical mobility, insufficient self-care, deficient knowledge, and deterioration of sleep pattern. The problems which were most frequently unresolved upon discharge of the patient from hospital were deterioration of urinary elimination, deficient knowledge, risk of unstable glucaemia and deterioration of sleep 
pattern. The most frequent medical complications were acute tubular necrosis and urinary fistula. Post-surgery blood transfusion was needed by $23 \%$ of the patients, $45 \%$ needed haemodialysis in the days after the transplant and $38 \%$ needed continuous vesicular irrigation.

\section{KEY WORDS: \\ KIDNEY TRANSPLANT \\ ELDERLY PATIENTS \\ COMPLICATIONS \\ NANDA TAXONOMY}

\section{Introducción}

El trasplante renal se ha convertido en la actualidad en el tratamiento de elección para pacientes con insuficiencia renal crónica terminal que precisan tratamiento sustitutivo. Mejora drásticamente la calidad de vida comparándolo con las otras terapias sustitutivas como la hemodiálisis y la diálisis peritoneal ${ }^{1,2}$. En los últimos años, se han producido dos fenómenos concurrentes como son, por una parte el aumento de la edad media de los donantes y por otro el incremento de la edad media de los receptores. En la Comunidad Autónoma Vasca se ha pasado de un promedio de edad de donante cadáver de 46,2 años en 1996 a 53,8 años en el 2002, según el informe de 2002 de Osakidetza Servicio Vasco de Salud ${ }^{3}$. Los donantes mayores de 65 años se han incrementado del $9 \%$ en el año 1996 al 36\% en el 2004. En esta Comunidad también se ha incrementado la edad promedio del enfermo en diálisis, pasando de 54 años en 1991 a 66 años en el 2004 y la de la lista de espera de trasplante renal, que ha pasado de 44 años a 51 años en el mismo periodo ${ }^{4}$, habiendo en la lista de espera una creciente inclusión de pacientes mayores de 60 años de edad. El número de trasplantes por grupos de edad realizados en nuestro centro desde 1995 al 2004, muestra que el grupo de edad que recibe más trasplantes es el de 45 a 59 años con un total de 479, y el segundo grupo es el de 60 a 74 años que recibió 330 injertos renales. En nuestro centro se comprobó también que la supervivencia del injerto procedente de donantes mayores de 65 años, se sitúa en torno al $65 \%$, por debajo de la supervivencia del injerto procedente de donantes menores de 65 años, que es del $77 \%$ a 5 años 5 .
El objetivo este trabajo fue aportar la visión de las complicaciones en el trasplante renal a pacientes añosos visto desde la óptica enfermera mediante la taxonomía NANDA6.

\section{Material y Métodos}

Se analizaron retrospectivamente las historias clínicas de trasplantados renales añosos, considerándose como tales a los mayores de 65 años en el momento de recibir el trasplante, que fueron intervenidos durante los años 2005 y 2006 en el Hospital de Cruces. El tiempo de seguimiento fue desde la realización del trasplante hasta su alta a domicilio. Se contabilizaron los diagnósticos de enfermería, su frecuencia y duración, así como las complicaciones médicas, número de transfusiones de sangre, sesiones de hemodiálisis e irrigaciones continuas vesicales post-quirúrgicas.

\section{Resultados}

El total de pacientes añosos trasplantados en ese periodo fue de 58 lo que supone el $22 \%$ del total de trasplantes realizados, el número de trasplantes realizados cada año y la edad de los receptores y donantes se refleja en la tabla 1 .

\begin{tabular}{|l|l|l|}
\hline $\mathbf{2 0 0 5}$ & Donantes & Receptores \\
\hline $\mathrm{N}^{0}$ & 24 & 27 \\
\hline EDAD & 68,04 & 68,15 \\
\hline Desv ST & 2,82 & 2,20 \\
\hline Rango & $65-73$ & $65-72$ \\
\hline 2006 & Donantes & Receptores \\
\hline $\mathrm{N}^{0}$ & 19 & 31 \\
\hline EDAD & 70,6 & 68,61 \\
\hline Desv. ST & 2,82 & 2,51 \\
\hline Rango & $66-74$ & $65-74$ \\
\hline
\end{tabular}

Tabla 1. Distribución de receptores añosos (> 65 años) de trasplante renal en los años 2005-2006

Por problemas logísticos hubo dos historias clínicas a las que no pudimos acceder por lo que presentamos los datos de 56 pacientes. De estos 56, 39 eran hombres ( $70 \%$ ) y 17 mujeres (30\%); 46 pacientes del total tenían entre 65 y 70 años (82\%) y 10 tenían entre 71 y 74 (18\%). Al alta del servicio de 
nefrología 53 pacientes tenían el injerto funcionante (95\%). De los 3 que no les funcionaba el injerto, 2 presentaban una necrosis tubular aguda severa y uno sufrió una trasplantectomía por hemorragia importante postquirúrgica.

En la actualidad, de los trasplantados en el 2005 el injerto permanece funcionante en el $85 \%$ de los casos (23). De los trasplantados en el 2006, aquellos que tienen un injerto funcionante son el $94 \%$ (29). Fallecieron 3 pacientes y se perdieron 3 injertos, 2 por rechazo crónico y 1 por trasplantectomía por sangrado postquirúrgico (figura 1). El promedio de estancia en el hospital fue de 22 días (rango7-75 días).

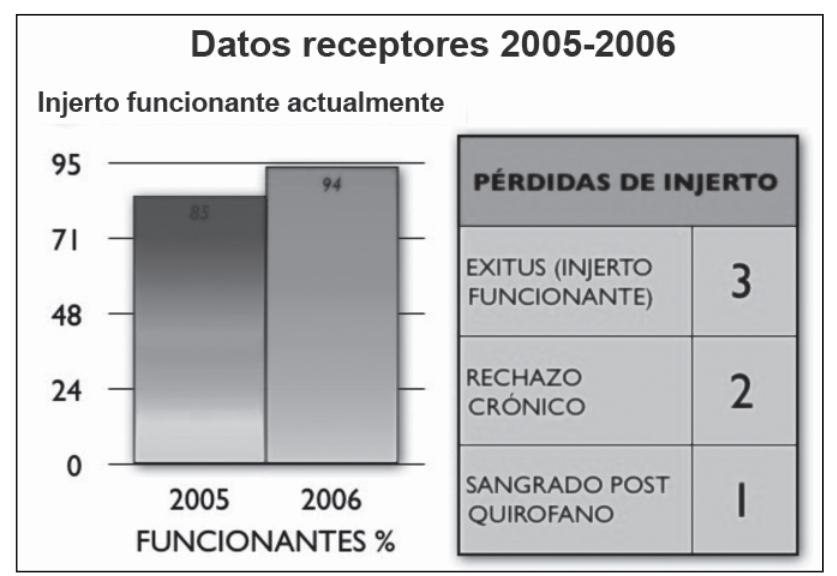

Figura 1. Injerto funcionante actualmente y causas de pérdida de injerto

La Unidad de Nefrología cuenta con 2 secciones de hospitalización: la $7^{\mathrm{a}}$ planta (agudos y URPA de Tx renal) y la $8^{a}$ planta (hospitalización) Los pacientes ingresan desde quirófano en la $7^{a}$ planta y permanecen en la unidad hasta su alta excepto en los casos de necesidad logística para la realización de nuevos trasplantes en cuyo caso son trasladados a la $8^{a}$ planta en habitaciones preparadas al efecto.

De los casos que nos ocupan 16 pacientes (29\%) fueron dados de alta desde la $7^{\mathrm{a}}$ planta con un promedio de estancia de 19 días $\pm 16,63$. El resto, 40 pacientes ( $71 \%$ ) fueron dados de alta desde la $8^{a}$ planta con una estancia promedio en esa planta de 14 días $\pm 8,01$.

Los Diagnósticos Enfermeros registrados en estos pacientes fueron:

- Riesgo de infección R/C procedimientos invasivos, inmunosupresión, alteración de las defensas primarias y de las defensas secundarias.
- Riesgo de lesión perioperatoria R/C factores externos e internos

- Exceso de volumen de líquidos R/C con el compromiso de los mecanismos reguladores y el exceso de aporte de líquidos.

- Deterioro de la integridad cutánea R/C la intervención quirúrgica.

- Deterioro de la movilidad física R/C con la prescripción de restricción de movimientos y el malestar o dolor.

- Déficit de autocuidados (baño/higiene, vestido/ acicalamiento, uso del WC) R/C debilidad y cansancio, dolor.

- Conocimientos deficientes respecto del proceso de trasplante renal y el régimen terapéutico $\mathrm{R} / \mathrm{C}$ limitación cognitiva.

- Insomnio R/C factores psicológicos, ambientales y fisiológicos.

- Estreñimiento R/C factores psicológicos, farmacológicos y fisiológicos.

- Riesgo de glucemia inestable R/C factores fisiológicos y farmacológicos.

- Perfusión tisular inefectiva renal R/C factores fisiológicos y farmacológicos.

- Dolor agudo R/C la intervención quirúrgica y obstrucciones del tracto urinario.

- Deterioro de la eliminación urinaria R/C multicausalidad.

- Hipertermia R/C enfermedad que afecta a la regulación de la temperatura.

- Retención urinaria R/C con obstrucción.

- Nauseas R/C con distensión gástrica causado por la administración de agentes anestésicos.

- Riesgo de déficit de volumen de líquidos R/C pérdidas excesivas por vías normales, uso de diuréticos.

- Ansiedad R/C el estado de salud.

- Diarrea R/C efectos adversos de los medicamentos.

- Deterioro del intercambio gaseoso R/C desequilibrio ventilación-perfusión y/o cambios en la membrana alveolar-capilar.

- Déficit de volumen de líquidos R/C Pérdida activa de volumen de líquidos y/o fallo de los mecanismos reguladores. 
- Confusión aguda R/C edad superior a los 60 años.

- Duelo complicado R/C pérdida real o percibida de un objeto (injerto renal).

La frecuencia con que se observaron los problemas más relevantes se recogen en la figura 2 .

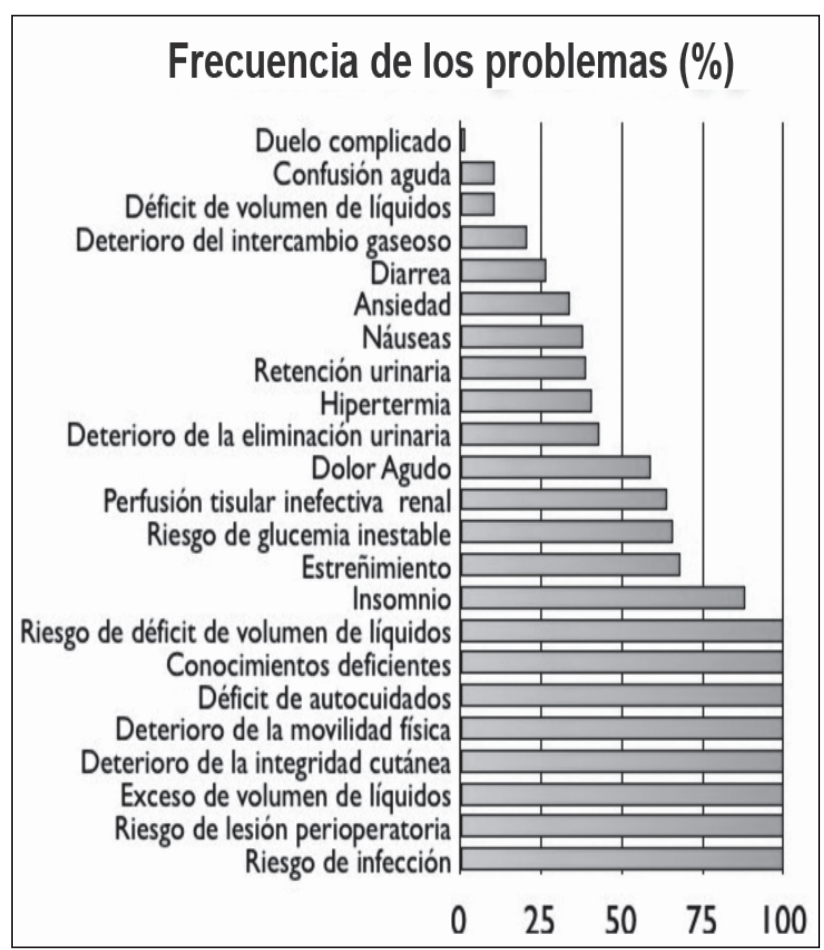

Figura 2. Frecuencia de los problemas de enfermería en porcentaje

Las complicaciones médicas halladas de mayor a menor frecuencia fueron:

- Necrosis Tubular Aguda: 21 pacientes (38\%) con una duración promedio de 13,70 días (rango 3- 40 días).

- Fístula urinaria: 7

-Problemas cardiacos: 5

- Bacteriemia: 4

- Dehiscencia de la herida quirúrgica: 3

- Hepatopatía: 3

- Estenosis ureteral: 3

- Infección urinaria: 3

- Rechazo agudo: 3

- Linfocele: 2

-Pancreatitis: 1

\section{- Leucopenia: 1}

- Hemorragia que precisó reintervención: 1

También se ha de destacar que 13 pacientes (23\%) precisaron transfusiones de sangre postcirugía y se transfundieron 31 unidades de concentrado de hematíes con un promedio por paciente transfundido de $2,38 \pm 0,77$ unidades. Por otra parte, 21 pacientes (38\%) precisaron de suero de lavado para tratar problemas de obstrucción de sonda vesical por hematuria y coágulos abundantes y 25 pacientes (45\%) precisaron sesiones de hemodiálisis por alteraciones hidroelectrolíticas en algún momento del post trasplante. Se realizaron 67 sesiones con un promedio por paciente de 2,68 sesiones.

\section{Discusión}

La identificación de los problemas de enfermería en el paciente trasplantado añoso mediante una taxonomía normalizada ofrece singulares ventajas al profesional como son la homogeneización de los criterios diagnósticos enfermeros, la claridad en el registro y la mejora en la planificación y evaluación de los cuidados de enfermería, permitiendo su investigación y la puesta en práctica de las estrategias de cuidados basadas en la mejor evidencia disponible. Los trabajos publicados se refieren fundamentalmente a los problemas de enfermería objetivados en el paciente trasplantado renal en tratamiento ambulatorio $^{7}$, por lo que es difícil establecer una comparación.

La correcta conceptualización e identificación de los problemas de enfermería es la base de la selección de las mejores intervenciones enfermeras para resolver o evitar problemas de salud. La taxonomía NANDA provee de unos criterios diagnósticos validados que son útiles en el quehacer enfermero. Aunque procede originalmente de los Estados Unidos ${ }^{8}$, otros autores defienden sus virtudes transculturales. La robustez de esa taxonomía se ha ido demostrando con el tiempo, por su extensión a otros países cómo Suecia, Gran Bretaña, Holanda o Islandia?.

Varios trabajos afirman que el trasplante renal es una opción válida para los pacientes renales añosos en tratamiento sustitutivo $0^{10,11,12,13}$, recomendando ajustar los donantes añosos a los receptores añosos ${ }^{14}$. 
En nuestro centro todos los pacientes con IRCT son considerados para ser incluidos en lista de espera de trasplante renal, a menos que existan contraindicaciones absolutas porque el trasplante ofrece mejor expectativa y calidad de vida que la diálisis. Se consideran contraindicaciones absolutas: el cáncer incontrolado, la infección activa sistémica y una expectativa de vida inferior a 2 años. Por lo tanto, no consideramos que la edad avanzada sea, en si misma, una contraindicación para el trasplante renal aunque en este caso es fundamental una valoración exhaustiva del estado cardiovascular.

El postoperatorio del trasplante no está exento de complicaciones ${ }^{15,16,17}$, además, en el caso de los donantes añosos los injertos renales son muy sensibles a cualquier agente nocivo tal como pueden ser la isquemia, el rechazo o los fármacos nefrotóxicos y se recuperan peor que los injertos más jóvenes ${ }^{18}$. Los receptores añosos tienen peores situaciones clínicas que los más jóvenes aunque el trasplante siga siendo su mejor opción a nivel general ${ }^{19}$

En nuestra serie la necrosis tubular aguda es la complicación médica más frecuente $(38 \%)$ mientras que otros trabajos con trasplantes de estas características mencionan un $31 \%^{20}$. Está acreditado que esta complicación está en relación con el tiempo de isquemia fría y que puede variar entre el $28 \%$ y el $35 \%{ }^{21}$ y otras series presentan cifras entre el $12 \%$ y el 43\% ${ }^{22}$. En Europa, existe el programa ESP (Europe Senior Programme) que se basa en localizar donantes de más de 65 años para receptores de las mismas características, intentando disminuir el tiempo de isquemia fría y mencionan una frecuencia de necrosis tubular aguda del 19\%, consiguiendo resultados tan buenos como los del programa habitual de trasplan$\mathrm{te}^{23}$, aunque su promedio de estancia es mayor: 32,2 $\pm 14,5$ días.

La dehiscencia de herida quirúrgica es una complicación poco frecuente (5\%).y otros autores en pacientes similares presentan cifras del $14 \%$. A pesar de en la bibliografía se citan hemorragias postquirúrgicas entre el $7 \%$ y el $11 \%$, en nuestra serie un paciente precisó de una trasplantectomía, aunque si se objetivaron anemizaciones importantes por sangrado postquirúrgico que precisaron transfusiones de sangre. Mucha de la literatura publicada hace referencia a periodos de seguimiento de un año. En nuestro caso, el periodo se limitó al tiempo de estancia post-trasplante con el fin de identificar los problemas de enfermería más comunes en ese periodo. Al ser un periodo tan reducido es difícil comparar los resultados con trabajos con periodos de seguimiento claramente mayores, aunque hay patologías como la necrosis tubular aguda, las fístulas urinarias, o la deshiscencia de herida quirúrgica que si pueden ser comparables.

\section{Conclusiones}

El trasplante renal en pacientes añosos es una opción viable que mejora la supervivencia del paciente aunque hay que seguir trabajando para mejorar las tasas de supervivencia de injerto y para reducir las complicaciones post-trasplante.

Las complicaciones médicas más frecuentes son la NTA, las anemizaciones postquirúrgicas que precisan transfusión y las fístulas urinarias durante su ingreso hospitalario postquirúrgico.

Los problemas de enfermería en el post trasplante renal más frecuentes son: riesgo de infección, riesgo de lesión perioperatoria, exceso de volumen de líquidos, deterioro de la integridad cutánea, deterioro de la movilidad física, déficit de autocuidados y conocimientos deficientes.

\section{Bibliografía}

1. Schnuelle P, Lorenz D, Trede M, Van der Moude FJ. Impact of renal cadaveric transplantation on survival in end-stage renal failure: Evidence for reduced mortality risk compared with haemodialysis during long-term follow-up. J Am Soc Nephrol 1998; 9:2135.

2. Snyder JJ, Kasiske BL, Gilbertson DT, Collins AJ. A comparison of transplant outcomes in peritoneal and hemodialysis patients. Kidney Int. 2002; 62:1423.

3. 2002. Informe de Coordinación de Trasplante. Osakidetza. Servicio Vasco de Salud.

4. Fuente: UNIPAR. Informe Epidemiológico de Pacientes Renales. 2004.

\section{UNIPAR, 2003}


6 NANDA. Diagnósticos enfermeros: definiciones y clasificación 2007-2008. Madrid: Elservier.

7 Lira, Ana Luisa Brandão de Carvalho; Albuquerque, Jaqueline Galdino; Lopes, Marcos Venícios de Oliveira. Perfil dos diagnósticos de enfermagem presentes em pacientes transplantados reanis. $\mathrm{R}$ Enferm UERJ 2007; 15(1):13-19.

8 Hogston R. Nursing diagnoses and classification systems: a position paper. Journal of Advanced Nursing. 1997; 26:496-500.

9 Thoroddsen A, Thorsteinsson H. Nursing diagnosis taxonomy across the Atlantic Ocean: congruence between nurses' charting and the NANDA taxonomy. Journal of Advanced Nursing. 2002; 37 (4): 372-381.

10. Orsenigo E, Casiraghi T, Socci C, Zuber V, Caldara $R$, Secchi A, Staudacher C. Impact of recipient and donor ages on patient and graft survival after kidney transplantation. Transplant Proc. 2007 Jul-Aug; 39(6):1830-2.

11. Nazemian F, Naghibi M, Farazi E. Kidney transplantation in elderly Iranian patients. Saudi J Kidney Dis Transpl. 2007 Jul-Sep; 18(3):391-6.

12. Pedroso $S$, Martins L, Fonseca I, Dias L, Henriques AC, Sarmento AM, Cabrita A. Renal transplantation in patients over 60 years of age: a single-center experience. Transplant Proc. 2006 Jul-Aug; 38(6):1885-9.

13. Foley DP et al. Long-term outcomes of kidney transplantation in recipients 60 years of age and older at the University of Florida. Clin Transpl. 2005; 101:9.

14. Kwon OJ, Lee HG, Kwak JY. The impact of donor and recipient age on the outcome of kidney transplantation. Transplant Proc. 2004 Sep; 36(7):2043-5.

15. Gea Caballero, V. Trasplante renal. Actualización y cuidados de enfermería. Enfermería Integral. 2000. 55: 7-14.
16. Moya Mora AM, Gómez I, Andugar Hernández J. Complicaciones post-quirúrgicas y secundarias a la medicación inmunosupresora del TX renal. Rev Soc Esp Enferm Nefrol. 1998 ene-mar; 1:27-33.

17. Berlango Jiménez J, Crespo Montero, R. Complicaciones del trasplante renal. En Andreu $L$ y Force E. La enfermería y el trasplante de órganos. Madrid: Editorial Médica Panamericana. 2004. p. 115-132.

18. Ferraresso M, Berardinelli L, Bellapi A, Vegeto A. Long-term function and survival rates of kidneys from extreme-age donors in the cyclosporine era. Transplant Proc. 1998 Aug; 30(5):2274-5.

19. Bentas W, Jones J, Karaoguz A, Tilp U, Probst M, Scheuermann E, Hauser IA, Jonas D, Gossmann $J$. Renal transplantation in the elderly: surgical complications and outcome with special emphasis on the Eurotransplant Senior Programme. Nephrol Dial Transplant. 2008 Jan; 18: 2043-2051.

20. Bertelli R et al. Bologna transplant centre results in double kidney transplantation: update. Transplant Proc. 2007 Jul-Aug; 39(6):1833-4.

21. Fría Ziaja J et al. Early donor lymph node procurement and local HLA typing reduce cold ischemia time and risk of acute tubular necrosis in cadaveric kidney transplantation. Transplant Proc. 2006 Jan-Feb; 38(1):39-41.

22. Giessing $M$ et al. Old-for-old" cadaveric renal transplantation: surgical findings, perioperative complications and outcome. Eur Urol. 2003 Dec; 44(6):701-8.

23. Fabrizii V, Kovarik J, Bodingbauer M, Kramar R, Hörl WH, Winkelmayer WC.Long-term patient and graft survival in the eurotransplant senior program: a single-center experience. Transplantation. 2005; 80(5):582-9. 